\title{
Timing noise analysis of pulsars: Tools and hidden gems
}

\section{Jacques Maritz ${ }^{* \dagger}$}

Physics Department, University of the Free State, 9300, South Africa

E-mail: maritzjmeufs.ac.za

\section{Pieter Meintjes}

Physics Department, University of the Free State, 9300, South Africa

E-mail: meint jpjeufs.ac.za

In this paper we review the timing noise phenomena seen in long-term timing of some pulsars and investigate the possible clock characteristics that can produce the observed quasi-periodic timing noise residual structures. We review some recent advances in analysis tools that can shed light on the connection between pulsar timing and possible timing noise processes.

Frontier Research in Astrophysics - II

23-28 May 2016

Mondello (Palermo), Italy

\footnotetext{
* Speaker.

${ }^{\dagger}$ The speaker wishes to acknowledge the SKA for financial support.
} 


\section{Introduction}

Pulsars are rapidly rotating, highly magnetized objects that are the remnants of supernovae explosions. These objects contribute to the fields of astrophysics, nuclear physics and experimental Radio Frequency astronomy, due to its high core density, fast spin-periods (e.g. [1]) and intricate pulse processing requirements. However, not all pulsars are observed as stable clocks, ultimately being unable to be fitted to the pulsar spin-down model. There exist a large number of factors that contribute to the "noise" reservoir observed in pulsars. These inaccuracies are broadly due to the clock mechanism itself, the mechanisms that produce the clock ticks (or pulses) in the magnetosphere, the interstellar medium (ISM) and some local reference clock effects (local time stamps).

The "noise" contribution due to the clock itself include glitches and spin-noise (variations in the spin-down rate of the pulsar), that produce an inaccurate predictions of the clock ticks. The "noise" induced by the ISM include frequency dependent dispersive delays, Faraday rotation and scattering due to irregularities in the ISM. The noise induced by the dynamics of the mechanism that produces the clock ticks includes profile evolution, both in frequency and time. Lastly, the local clock effects include the "noise" contribution from the ionosphere, polarization instrumental leakage, radiometer noise of the system and the precision levels in planetary ephemerides that are used to convert the times of arrival (TOA) to the solar system barycenter.

After a few observations the astronomer can start to determine the phase-connected solution of the pulsar, after which we can predict the TOA of the pulses and predict what the pulsar's spin frequency will be at any given time. A phase connected solution allows us to measure the pulsar's spin, astrometric parameters and orbital parameters. Pulsar timing represents the technique of comparing the observed and computed pulse TOA (or clock ticks). This comparison produces residuals that are non-zero and are more commonly known as timing noise given that the noise spectrum is red. Timing noise can be considered as random variations in the spin-down rate of the pulsar with time scales of months to years, particularly seen in slow-spinning pulsars but also in millisecond pulsars ([2]).

Timing noise structures in timing residuals were already analyzed in the mid-nineties for several southern pulsars ([3]), clearly showing that the power density spectra of the phase residuals follow some red-noise type frequency dependence, that is $f^{-\alpha}$. These spin-down rate changes can be very small (less than one percent), with the exception of large mass accretion rates due to orbiting bodies ([4]). Any systematic change, that rules out the possibility of the above mentioned noise contributions, in the spin-down rate could imply some change in the Goldreich-Julian charge density in the open field regions, implying that the change in spin-down rate and changes in the measured profile can be correlated in some way, possibly explaining the origin of the observed pulsar timing noise, i.e. timing noise can either be due to changes in the clock mechanism itself (changes in the magnetosphere) or external torque mechanisms.

Most of the noise processes in timing can be whitened (or removed), but a careful analysis of the observed timing noise can still reveal some interesting models that can describe the random variations observed in the spin-down rate. In the following sections we review the possible tools and models that can be used to extract the spin-down rate by using the timing noise residuals and TOA errors of pulsars. 


\section{Tools}

Timing noise can be realized in the measurement of the second derivative of the spin frequency,

$$
\ddot{v}=\frac{n \dot{v}^{2}}{v},
$$

where $n=3$ if we assume the dipole spin-down model, which is hardly ever the case. If we use a pulsar with spin-period of $P \approx 500 \mathrm{~ms}$, or $v \approx 2 \mathrm{~Hz}$, together with $n=3$ and a first frequency derivative of $\dot{v} \approx 10^{-14} \mathrm{~s}^{-2}$, we get the value for the second derivative of the spin frequency as $\ddot{v} \approx 10^{-28} \mathrm{~s}^{-3}$. Apart from some young pulsars, the value for $\ddot{v}$ is larger than predicted for the $n=3$ spin-down model, clearly indicating the presence of timing noise.

To search for possible time scales involved in the spin-down behavior one can use the nonzero residuals, the TOA errors and the fact that the residual pattern is locally predictable, in order to predict, using the most unbiased method possible, the evolution of the spin-down of a particular pulsar ([4]). One can derive $v$ and $\dot{v}$, by inspecting the residual pattern piece by piece and using the glitch plugin that is part of TEMPO2 ([5]). This plugin was engineered to look for glitches in timing residuals that will show evident changes in both $v$ and $\dot{v}$. However, from the statistics point of view, partitioning residuals (e.g. selecting residual windows with length of 300 days) that contain irregular observation cadences, could induce biases seen in $v$ and $\dot{v}$.

It is more beneficial to fit an analytic function to the residuals that statistically deals with the TOA errors and the quasi-periodicity of the residual structure. We start to realize the time series of the residuals in the form $\mathbf{y}=f(\mathbf{x})+\zeta$, where $\zeta$ represents the noise in the model. To infer from the time series, we need to learn the function $f$ and the probability distribution of $y_{n}$ given $x_{n}$, that is $p\left(y_{n} / x_{n}\right)$ that can be used to find the parameters that best fit the system.

We use the process of curve fitting for this problem, since we can assume that $\mathbf{y}$ is ordered by $\mathbf{x}$, which can be seen by closely looking at the quasi-periodic structure seen in the timing noise residuals. We can construct a co-variance kernel, $k\left(x_{i}, x_{j}\right)$, that describe the connection between any points, together with the fact that the observations do not have to lie on a predefined distribution, making it truly unbiased. To perform the curve fitting, we initialize the squared exponential (or any other kernel that describes the data set) and repeatedly infer over many distributions until we statistically find the best curve that describes our time series, i.e. we can learn the best mean and variance for our curve fitting. We can now find any $y\left(x_{n}\right)$, see Fig. .

It is clear from the example above that the curve fitting will be more efficient if more data points are used in the time series. The error bands increase for the start and end of the curve fitting since the Gaussian process depends on future/past points to find the best prior, moreover the time series is sparsely populated for the first/last part.

\section{Hidden gems}

By looking at the variations in the spin-down rate of some pulsars, several authors were led to investigate the possible correlation of the spin-down variations (calculated using the either TEMPO2 of Gaussian curve fitting) with any changes in the clock mechanism, that is, changes in the emission mechanisms itself (e.g. see [5]) . This can be done by searching for profile variations, both temporally or by searching for changes in the polarization state of the pulse itself. 


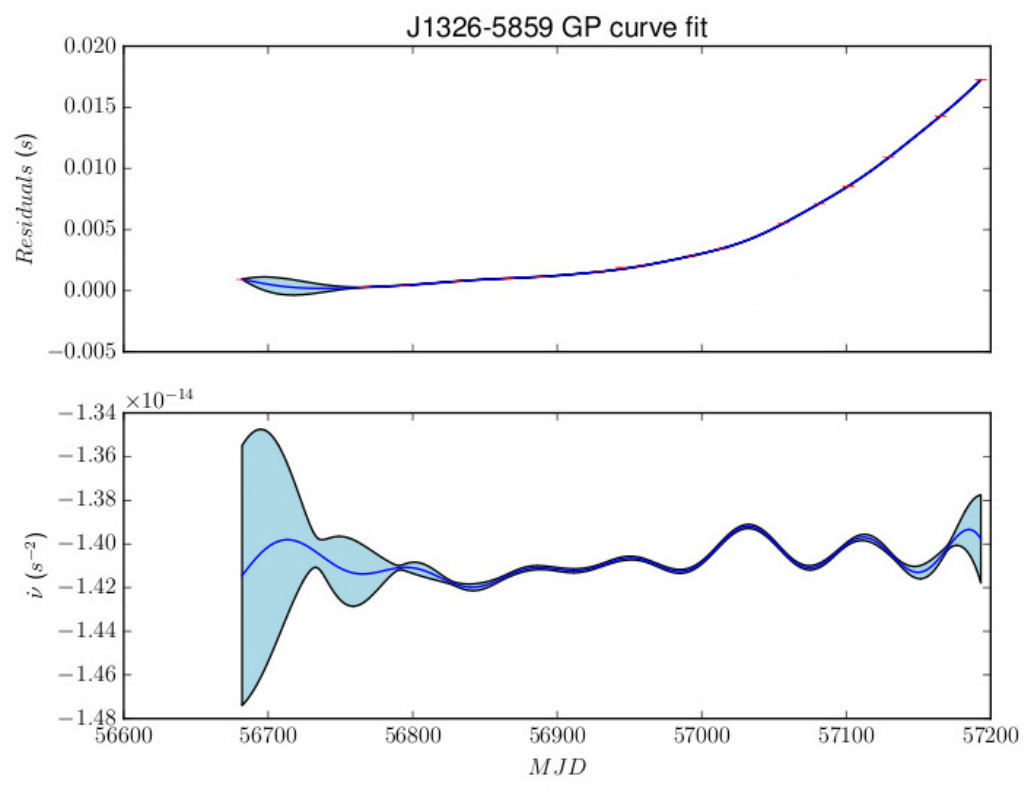

Figure 1: Example of Gaussian process curve fitting for PSRJ1326-5859 using the code described in [6], data retrieved from the Parkes pulsar archive as part of the timing campaign described in [7].

Searching for correlations using profile variations depends largely on a sufficient amount of profiles spread over the time range of interest. These profiles need to be phase aligned and exhibit a $\mathrm{S} / \mathrm{N} \geq 10$, since the analysis is phase bin resolved and more complex kernels are needed to fit a time series of low $\mathrm{S} / \mathrm{N}$ pulses ([8]). In the quest to correlate emission with rotation, one handy tool could be to track the polarization vector of a specific pulse component over the range of interest. Oslowski et al[9] and references there-in already pioneered this technique in the search for orthogonal polarization modes that can be seen as part of a phase-resolved single pulse polarization study of PSR J0437-4715. We performed a similar analysis on the main pulse component of a single $3100 \mathrm{MHz}$ pulse of PSR J1326-5859 and noticed that only one mode of emission is present, see Fig.[2.

Probably one of the most famous applications of the emission-rotation correlation search technique, is the case of the PSR J0738-4042 encountering an asteroid ([4]), see Fig. []. Pulse drifting with respect to the change in spin-down rate indicated a correlation between emission and rotation, and using the change in the spin-down rate the conclusion was reached that the pulsar interacted with a billion ton asteroid.

\section{Conclusions}

In this paper we reviewed some of the tools and gems of timing noise analysis, particularly emphasizing statistical curve fitting techniques for emission-rotation correlation in both the profile and spin-down rate domains. However, the origins of timing noise still remains unclear and causes imperfections in our clocks over decades of timing. 


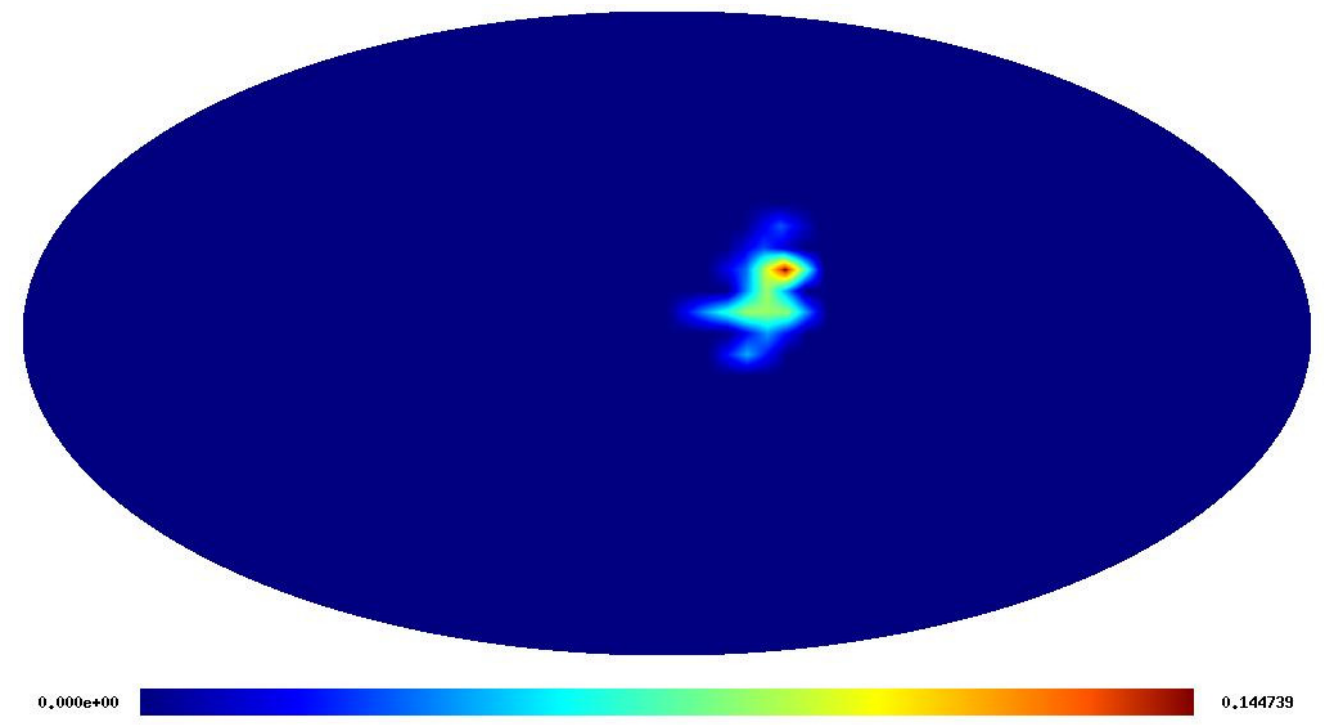

Figure 2: Aitoff-Hammer projection of the phase-resolved polarization vector for the main component of a $3100 \mathrm{MHz}$ pulse of PSR J1326-5859.

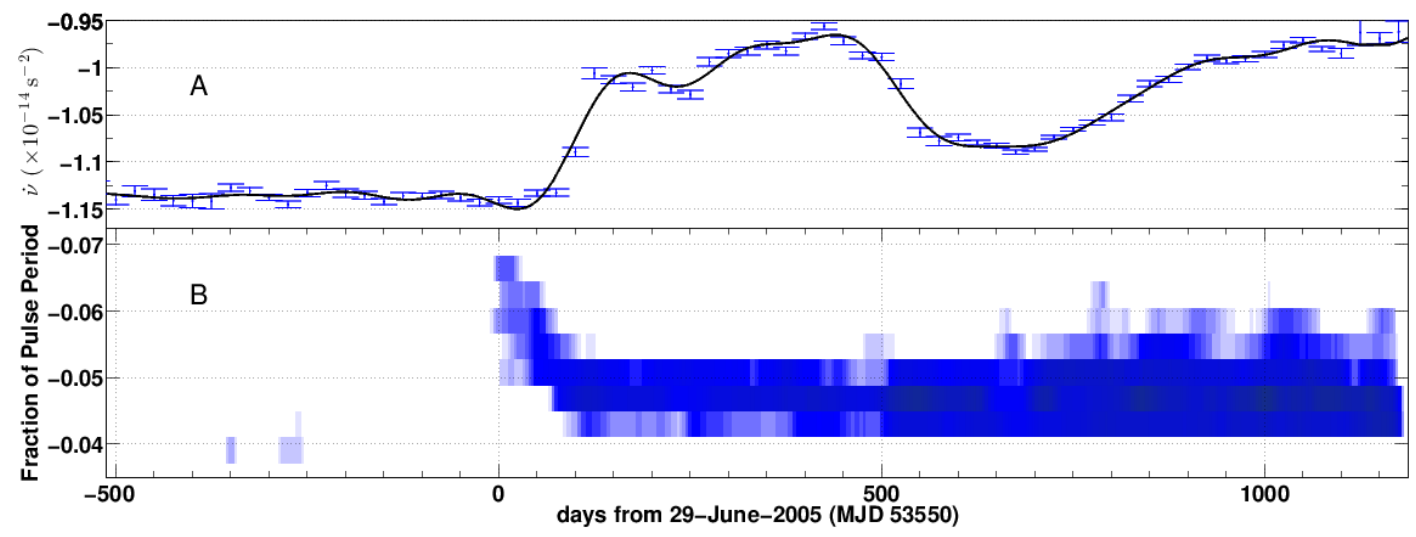

Figure 3: Example of searching for emission-rotation correlations for PSR J0738-4042. Panel A indicates the computed spin-down rate and panel B illustrates bin-resolved profile residuals. Adopted from [4].

Perhaps more emphasis should be placed on a model that can explain both observed emissionrotation correlations and anti-correlations that is unique to a pulsar and its place on the $\dot{P}-P$ diagram. Typically this model can be in the form a disk (mixture of gaseous and rock material) extending partially across the co-rotation radius of the pulsars. These disks can be in the form of supernovae fall-back or debris swept up by the pulsar moving through a field polluted with planetesimals. The magnetic field threads the disk in this scenario and the disk exerts a total torque on the pulsar in the form of magnetic torques and the propeller effect, ultimately changing its braking index and spin-down rate. However, measuring braking indexes in pulsars exhibiting timing noise remains tricky, and the observational evidence of pulsar-disk interactions need to be found by exploring 
other avenues.

\section{References}

[1] D. R. Lorimer. Handbook of pulsar astronomy. Cambridge University Press, Cambridge, UK New York, 2005. ISBN 978-0-521-82823-9.

[2] P. D. Lasky, A. Melatos, V. Ravi, and G. Hobbs. Pulsar timing noise and the minimum observation time to detect gravitational waves with pulsar timing arrays. Monthly Notices of the Royal Astronomical Society, 449:3293-3300, May 2015. doi: 10.1093/mnras/stv540.

[3] F. D'Alessandro, A. A. Deshpande, and P. M. McCulloch. Power Spectrum Analysis of the Timing Noise in 18 Southern Pulsars. Journal of Astrophysics and Astronomy, 18:5-14, March 1997. doi: 10.1007/BF02714849.

[4] P. R. Brook, A. Karastergiou, S. Buchner, S. J. Roberts, M. J. Keith, S. Johnston, and R. M. Shannon. Evidence of an Asteroid Encountering a Pulsar. ApJL, 780:L31, January 2014. doi: 10.1088/2041-8205/780/2/L31.

[5] G. Hobbs, R. Edwards, and R. Manchester. Tempo2: a new pulsar timing package. CJAA, 6 (2):189, 2006.

[6] M. Seikel, C. Clarkson, and M. Smith. Reconstruction of dark energy and expansion dynamics using gaussian processes. JRASC, 6:036, 2012.

[7] R. N. Manchester, G. Hobbs, M. Bailes, W. A. Coles, W. van Straten, M. J. Keith, R. M. Shannon, N. D. R. Bhat, A. Brown, S. G. Burke-Spolaor, D. J. Champion, A. Chaudhary, R. T. Edwards, G. Hampson, A. W. Hotan, A. Jameson, F. A. Jenet, M. J. Kesteven, J. Khoo, J. Kocz, K. Maciesiak, S. Oslowski, V. Ravi, J. R. Reynolds, J. M. Sarkissian, J. P. W. Verbiest, Z. L. Wen, W. E. Wilson, D. Yardley, W. M. Yan, and X. P. You. The Parkes Pulsar Timing Array Project. Publications of the Astronomical Society of Australia, 30:e17, January 2013. doi: 10.1017/pasa.2012.017.

[8] P. R. Brook, A. Karastergiou, S. Johnston, M. Kerr, R. M. Shannon, and S. J. Roberts. Emission-rotation correlation in pulsars: new discoveries with optimal techniques. Monthly Notices of the Royal Astronomical Society, 456:1374-1393, February 2016.

[9] S. Osłowski, W. van Straten, M. Bailes, A. Jameson, and G. Hobbs. Timing, polarimetry and physics of the bright, nearby millisecond pulsar PSR J0437-4715 - a single-pulse perspective. Monthly Notices of the Royal Astronomical Society, 441:3148-3160, July 2014. doi: 10.1093/mnras/stu804. 